\title{
Risk factors of hemorrhagic and ischemic stroke among hospitalized patients in Bangladesh - A case control study
}

\author{
Riaz BK ${ }^{1}$, Chowdhury $\mathrm{SH}^{2}$, Karim $\mathrm{MN}^{3}$, Feroz $\mathrm{S}^{4}$, Selim $\mathrm{S}^{5}$, Rahman $\mathrm{MR}^{1}$ \\ 1. NIPSOM, Dhaka, 2. Department of Medicine, Sarkari Kormachari Hospital, Dhaka, 3. WHO, Dhaka, \\ 4 Department of Medicine, Shaheed Suhrawardy Medical College Hospital, Dhaka, \\ 5. Department of Endocrinology, BIRDEM, Dhaka, \\ Email: selimshahjada@gmail.com
}

\begin{abstract}
The risk factor profiles, management and outcome have significant difference between stroke subsets. Aim of this study was to investigate the risk for the two most common subtypes of stroke in Bangladeshi population. Seventy cases of hemorrhagic stroke (HS) and 105 cases of confirmed ischemic stroke (IS) were recruited from the Shaheed Suhrawardy Medical College Hospital (ShSMCH) and Dhaka Medical College Hospital between January-June 2011. Total 171 age, sex matched controls were selected from the hospitalized patients with history of no stroke ever. Average hemorrhagic stroke patients $(60.4 \pm 12.3$ years) were younger than both ischemic strokes $(63.5 \pm 13$ years). Family history of premature cardiovascular death was found more in HS patients ( $\mathrm{p}=0.001)$. Multivariate logistic regression showed, in IS model 'less fruit consumption (OR 4.6), table salt intake (OR 8.15), psychosocial stress (OR 3.5), abnormal ECG (OR 3.6) and Increased WHR (OR 6.9) appeared as significant predictors adjusted for all potential candidate confounders. In HS model less fruit consumption (OR 5.0), table salt intake (OR 9.9), Stress (OR 4.1), family history of cardiovascular disease (CVD) death (OR 11.3), hypertension (OR 43), aspirin intake (OR 4.5) and increased WHR (OR 3.7) remained as significant predictors.
\end{abstract}

\section{Introduction}

Stroke is a leading cause of disabling morbidity and death in many countries of the world, ${ }^{1,2}$ Stroke is the second leading cause of death worldwide, and the leading cause of acquired disability in adults in most regions, ${ }^{3,4}$ In Asia, the problem of stroke has a particularly strong impact, not only because more than half of the world's population lives in Asia, but stroke is the predominant vascular disease in many parts of Asia. ${ }^{5}$ The prevention, identification and control of the cardiovascular risk factors at individual and population level is poor in Asia because of ignorance and high cost of the interventions. The burden of stroke is likely to increase substantially in the near future because of the aging population. Moreover, the financial burden of stroke on society is high, including both direct and indirect costs for stroke care. ${ }^{6}$ In a hospital based study, it was revealed that hypertension is the most important risk factor for stroke in Bangladesh. ${ }^{7}$ The identification of locally prevalent risk factors associated with different types of strokes [ischemic (IS) and hemorrhagic (HS)] may help the national control program to prioritize prevent/control strategies to reduce the burden of stroke in a country specific way.
Since treatment measures for stroke are still rather limited and expensive, in a high prevalent country like Bangladesh, it is important to be familiar with relative contribution of different stroke risk factor in an individual patient. The individuals with a relatively high risk profile can take steps to modify their risk factors through lifestyle changes and/or medical treatment. Similarly, public awareness programs aimed at increasing the recognition of stroke warning signs and altering modifiable risk factors can be designed to address the high-risk groups. Ischemic stroke is caused by obstruction of extra- or intracranial blood vessels, whereas hemorrhagic stroke is caused by rupture of intracranial blood vessels. HS are considered to have a higher mortality risk than IS. ${ }^{8}$ Previous studies have linked the excess mortality to the generally more severe strokes in patients with HS, whereas stroke type was not considered to be associated with mortality, ${ }^{9,10}$ Numbers in these studies were, however, few. Some risk factors are common for both HS and IS, ${ }^{11,12}$ and there are some factors that favors either type and some favors both. Badiuzzaman et al., found that among admitted stroke patients highest number $(56.25 \%)$ had 
cerebral infarct followed by cerebral hemorrhage $(38.25 \%){ }^{13}$ Knowledge on the relative contribution of risk factors in hemorrhagic vs ischemic strokes is still insufficient. Thus, the aim of this study was to investigate the risk for the two most common subtypes of stroke in Bangladeshi population by case-control study design which may give an insight to the clinicians in managing and preventing the events.

\section{Material and Methods}

A tertiary level hospital based case control study recruited 70 cases of hemorrhagic stroke (HS) and 105 cases of ischemic strokes (IS) confirmed by neuro-imaging (CT or MRI scan of Brain) from the ShSMCH and Dhaka Medical College Hospital in Dhaka between Jan-Jun 2011. Sample size was determined by STATAR, for comparison proportion of hypertension (one of the most striking risk factors in stroke) was considered. To detect odds ratio $>3$, with the proportion exposed among controls of 0.15 and the proportion exposed among cases of 0.340 , at $80 \%$ power and with number of control three fold of case a total of 70 cases of hemorrhagic stroke and 105 ischemic stroke is required. All the available and eligible cases during the study period from Medicine and Gynecology and Obstetric departments were taken as control.

Subjects with acute stroke and focal neurological deficit lasting $<24$ hours, death within 24 hours of admission, past H/O stroke, primary sub-arachnoid hemorrhage, co-existing acute coronary syndrome and unwilling to participate were excluded from the study. The primary distinction between stroke subtypes-ischemic, intra-cerebral hemorrhagic, and subarachnoid hemorrhagic — was based on neuro-imaging (CT or MRI). After obtaining written informed consent from the patient or the closest attendant, a semi-structured questionnaire was administered, which included information on all the stroke risk factors. The variables included to record/measure were history of hypertension and diabetes mellitus, physical activity, diet and alcohol intake, smoking status, non-smoke tobacco consumption and psychosocial factors, blood pressure and heart rate, and anthropometrical measurements (waist and hip circumference, height, and weight). Waist and hip were measured according to the United States National Institute of Health Practical Guide to Obesity. ${ }^{9}$ We assessed both waist-hip ratio and body-mass index as risk factors. We defined diabetes as having a fasting plasma glucose level of $>7 \mathrm{mmol} / \mathrm{L}(126 \mathrm{mg} / \mathrm{dL})$, a random glucose level of $>11.1 \mathrm{mmol} / \mathrm{L}$ (200 $\mathrm{mg} / \mathrm{dL}$ ), or the requirement of regular hypoglycemic drugs. Individuals were classified as physically active if they were regularly involved in moderate exercise (walking, cycling, or gardening) or strenuous exercise (jogging, football playing, and vigorous swimming) for four hours or more per week.

Smoking status was defined as never, former, or current smoker. We defined current smokers as individuals who smoked any tobacco in the past one month and included those who had quit within the preceding month. Former smokers were defined as those who had quitted more than a month earlier. Fruit consumption was defines as adequate if they reported to consume five or more serving of intake per day. Depression was defined as feeling sad, blue, or depressed for two or more consecutive weeks during the past 12 months. Atrial fibrillation or flutter was based on self-reported history, review of baseline ECG. Abnormal ECG was defined as having any abnormality diagnosed by a cardiologist. The same definitions were used for the controls, who were recruited from the hospitalized patients admitted to units other than medicine and never had a stroke in the past.

Age and sex for the controls were matched. Data were entered, cleaned and analyzed using STATA 10 software. For background variables and sociodemographic data descriptive statistics and relative frequency were generated. The data for each case and its matching control(s) were initially reviewed using STATA 10 software for tabulation of descriptive statistics. Individual risk factors were assessed for both stroke type adjusting for age and sex. Adjusted Odds Ratio with 95\% CI was assessed generating binary logistic regression model adjusting for all possible confounders.

\section{Result}

A total of 171 controls, 70 patients with HS and 105 patients with IS were recruited during the study period. On average Hemorrhagic (60.4 \pm 12.3 years) stroke patients were younger (not significant) than both Ischemic strokes $(63.5 \pm 13$ years $)$ and healthy controls (63.7 \pm 9.4 years). Proportion of males was also similarly high in all three comparison groups (p>0.05). Poor socio-economic status (Family earning <30000/ month) was associated with more $(p=0.02) \quad$ IS's. Family history of premature cardiovascular death was found to be associated with HS $(\mathrm{P}=0.001)$ (Table-I). 
Table I: Socio demographic factors of stroke

\begin{tabular}{|c|c|c|c|}
\hline & Control & Ischemic & Hemorrhagic \\
\hline \multicolumn{4}{|l|}{ Age } \\
\hline$<60$ years & $58(33.9 \%)$ & $35(33.3 \%)$ & $29(41.4 \%)$ \\
\hline$\geq 60$ years & $113(66.1 \%)$ & $70(66.7 \%)$ & $41(58.6 \%)$ \\
\hline Test statistics & Referent & $\chi^{2} 0.01$ p .921 & $\chi^{2} 1.2$ p 0.270 \\
\hline \multicolumn{4}{|l|}{$\operatorname{sex}$} \\
\hline Male & $125(73.1 \%)$ & $80(76.2 \%)$ & $45(64.3 \%)$ \\
\hline Female & $46(26.9 \%)$ & $25(23.8 \%)$ & $25(35.7 \%)$ \\
\hline Test statistics & Referent & $\chi^{2} 0.5$ p 0.568 & $\chi^{2} 1.9$ p 0.173 \\
\hline \multicolumn{4}{|l|}{ Religion } \\
\hline Muslim & $162(94.7 \%)$ & $92(87.6 \%)$ & $65(92.9 \%)$ \\
\hline Hindu & $9(5.3 \%)$ & $13(12.4 \%)$ & $5(7.1 \%)$ \\
\hline Test statistics & Referent & $\chi^{2} 4.4$ p 0.034 & $\chi^{2} 0.3$ p 0.571 \\
\hline \multicolumn{4}{|l|}{ Monthly Income } \\
\hline $\begin{array}{l}\geq 30,000 \\
\text { BDT/month }\end{array}$ & $101(59.1 \%)$ & $47(44.8 \%)$ & $39(55.7 \%)$ \\
\hline $\begin{array}{l}\leq 30,000 \\
\text { BDT/month }\end{array}$ & $70(40.9 \%)$ & $58(55.2 \%)$ & $31(44.3 \%)$ \\
\hline Test statistics & Referent & $\chi^{2} 5.4$ p 0.021 & $\chi^{2} 0.3 p 0.632$ \\
\hline \multicolumn{4}{|l|}{ Family size } \\
\hline$<5$ members & $124(72.5 \%)$ & $72(68.6 \%)$ & $49(70.0 \%)$ \\
\hline$\geq 5$ members & $47(27.5 \%)$ & $33(31.4 \%)$ & $21(30.0 \%)$ \\
\hline Test statistics & Referent & $\chi^{2} 0.5$ p 0.483 & $\chi^{2} 0.2$ p 0.694 \\
\hline \multicolumn{4}{|l|}{$\begin{array}{l}\text { Family } H / O \\
C V D \text { death }\end{array}$} \\
\hline No & $169(98.8 \%)$ & $103(98.1 \%)$ & $63(90.0 \%)$ \\
\hline $\begin{array}{l}\text { Yes } \\
\text { Test statistics }\end{array}$ & $\begin{array}{l}2(1.2 \%) \\
\text { Referent }\end{array}$ & $\begin{array}{c}2(1.9 \%) \\
\chi^{2} 0.3 \text { p } 0.620\end{array}$ & $\begin{array}{c}7(10.0 \%) \\
\chi^{2} 10.8 \mathrm{p} 0.001\end{array}$ \\
\hline
\end{tabular}

Age and sex adjusted risk factor for IS and HS

Table-II tabulated the age and sex adjusted risk factors for IS and HS generated individual logistic regression. Increased waist-to-hip ratio (OR 5.5), table (extra) salt intake (OR 5.6), low fruit consumption (OR 3.5 2.1-5.9), dyslipidemia (OR 2.3) appeared as significant predictor for ischemic stroke.

For hemorrhagic stroke a similar set of variables appeared to predict the disease. Increased waist-tohip ratio (OR 3.5), table salt intake (OR 4.6), low fruit consumption (OR 4.7, 2.5-8.6), stress (OR 4.1), smokeless tobacco use (OR 3.2), also appeared as significant predictor for HS. Additionally frequent fatty diet intake (OR 2.2), betel nut chewing habit (OR 2.5) and history of aspirin intake (OR 4.7) were found be significant predictors of HS.

Separate Multivariate logistic regression model were generated for predicting Ischemic and hemorrhagic stroke adjusted for all possible candidate predictors pooled from univariate analysis.

In IS model 'less fruit consumption (OR 4.6), table salt intake (OR 8.15), psychosocial stress (OR 3.5), abnormal ECG (OR 3.6) and Increased WHR (OR 6.9) remained as significant predictors adjusted for all potential candidate confounders. The model explained $52.5 \%$ of the variation in disease occurrence (Nagelkerke R Square 0.525) (Table III).

In HS model 'less fruit consumption (OR 5.0), table salt intake (OR 9.9), Stress (OR 4.1), Family history of CVD death (OR 11.3), Hypertension (OR 43), aspirin intake (OR 4.5) and increased WHR (OR 3.7) remained as significant predictors adjusted for all potential candidate confounders. The model explained $52.5 \%$ of the variation in disease occurrence (Nagelkerke R Square 0.715) (Table - III).

Table II: Odds ratio $(95 \% \mathrm{CI})$ for potential risk factors adjusting for age and sex

\begin{tabular}{lllll}
\hline Variable & \multicolumn{2}{l}{ Ischemic stroke } & \multicolumn{2}{l}{$\begin{array}{l}\text { Hemorrhagic } \\
\text { stroke }\end{array}$} \\
& OR & $95 \%$ CI & OR & $95 \%$ CI \\
\hline Low physical activity & 1.3 & $0.8-2.2$ & 0.7 & $0.4-1.2$ \\
Raised waist-to-hip ratio & 5.5 & $2.8-10.8^{*}$ & 3.5 & $1.7-7.3^{*}$ \\
Salt consumption & 5.6 & $3.3-9.6^{*}$ & 4.6 & $2.5-8.5^{*}$ \\
Frequent fatty diet & 1.5 & $0.9-2.6$ & 2.2 & $1.2-4.2^{*}$ \\
Low fruit consumption & 3.5 & $2.1-5.9^{*}$ & 4.7 & $2.5-8.6^{*}$ \\
Current smoker & 1.9 & $1.1-3.4$ & 1.9 & $0.9-3.8$ \\
Smokeless tobacco & 2.7 & $1.6-4.8^{*}$ & 3.2 & $1.7-6.1^{*}$ \\
Betel but chewing & 1.9 & $1.1-3.2$ & 2.5 & $1.3-4.7^{*}$ \\
Abnormal ECG change & 3.1 & $1.7-5.7^{*}$ & 0.7 & $0.3-1.9$ \\
Hypertension & 1.8 & $1.1-3.2$ & 21.0 & $9.9-45.5^{*}$ \\
Diabetes Mellitus & 0.8 & $0.4-1.4$ & 1.3 & $0.7-2.6$ \\
Dyslipidaemia & 2.3 & $1.1-4.8^{*}$ & 0.9 & $0.3-2.6$ \\
Aspirin intake & 2.3 & $0.9-5.5$ & 4.7 & $1.9-11.1^{*}$ \\
\hline
\end{tabular}

Model for predicting Ischemic and hemorrhagic stroke

Table III: Multiple regressions model for predicting Ischemic and hemorrhagic stroke

\begin{tabular}{|l|c|c|}
\hline Multiple regressions models & OR & $95 \%$ C.I. \\
\hline Ischemic stroke risk model & & $0.92-3.6$ \\
\hline Low physical activity & 1.81 & $2.32-9.16^{*}$ \\
\hline $\begin{array}{l}\text { Fruit consumption < } \\
\text { servings/day }\end{array}$ & 4.62 & $3.95-16.8^{*}$ \\
\hline Table salt intake & 8.15 & $1.73-7.10^{*}$ \\
\hline Psychosocial stress & 3.51 & $0.92-4.27$ \\
\hline Smokeless tobacco use & 1.98 & $1.49-8.53^{*}$ \\
\hline Abnormal ECG change & 3.57 & $0.92-9.71$ \\
\hline Aspirin intake & 2.99 & $3.00-15.95^{*}$ \\
\hline Increased Waist-to-hip Ratio & 6.92 & $95 \%$ C.I. \\
\hline Hemorrhagic stroke risk model & OR & $1.99-12.72^{*}$ \\
\hline $\begin{array}{l}\text { Fruit consumption < 5 } \\
\text { servings/day }\end{array}$ & 5.02 & $3.32-29.60^{*}$ \\
\hline Table salt intake & 9.91 & $1.55-81.58^{*}$ \\
\hline Family history of CVD death & 11.25 & $1.54-11.09^{*}$ \\
\hline Psychosocial stress & 4.14 & $0.47-1.28$ \\
\hline Abnormal ECG change & 0.68 & $13.9-132.5^{*}$ \\
\hline Hypertension & 42.87 & $1.25-11.16^{*}$ \\
\hline Aspirin intake & 4.45 & \\
\hline Increased Waist-to-hip Ratio & 3.74 & \\
\hline
\end{tabular}




\section{Discussion}

Higher waist-to-hip ratio, table salt intake, psychosocial stress, and low fruit consumption appeared as significant predictor for both Hemorrhagic and Ischemic stroke. Similarly hypertension, family history of CVD death and history of aspirin intake contributed to HS, but not with IS. In general, the stroke risk factors irrespective of the subtype are similar to the previous studies from home and abroad and the global prevention and control measures are to be undertaken for national stroke prevention strategy for Bangladesh.

The risk factor profiles of stroke subtypes differ, reflecting their differing etiopathology. It is important to distinguish subjects with risk of hemorrhagic stroke from ischemic subtype. In a population-based case-controlled study, hypertension and diabetes favored IS and high alcohol intake favored HS, whereas smoking did not favor either of the stroke subtypes. ${ }^{14}$ In another population-based observational study ${ }^{15}$ increasing age, previous stroke, and diabetes favored IS, whereas ischemic heart disease, atrial fibrillation, hypertension, alcohol intake, and smoking did not favor either of the stroke subtypes. In the hospitalbased Copenhagen Stroke Study ${ }^{14}$ diabetes and ischemic heart diseases favored IS, whereas age, hypertension, alcohol consumption, atrial fibrillation, and smoking were not predictors of stroke subtype. In the hospital-based Lausanne Stroke registry ${ }^{16}$ smoking, hypercholesterolemia, migraine, previous transient ischemic attack, atrial fibrillation, and heart disease favored IS, whereas hypertension was the only significant factor related to HS. When individual factors are tested after adjustment for age and sex, factors like higher waist-to-hip ratio, salt consumption, low fruit consumption, psycho social stress, smokeless tobacco, betel nut chewing, abnormal ECG change and dyslipidemia favored ischemic stroke. Surprisingly established factors of cerebrovascular disease like low physical activity, frequent fatty diet, current smoking, hypertension and diabetes mellitus did not show any association. Non association of aspirin is however understandable for ischemic stroke. A similar battery of factors appeared to be associated with hemorrhagic stroke risk and they are raised waist-to-hip ratio, salt consumption, frequent fatty meal, low fruit consumption, psycho social stress, smokeless tobacco use, betel nut chewing, hypertension and aspirin intake.

The possible co-linearity between factors might have contributed to the non association of few obvious factors like smoking, low physical activity etc. The study attempted to explore to possible factors of strokes of different types. Such selection of variables, leaves the ground for effect modification. Such interaction between variables confirms the clustering phenomena of noncommunicable disease risk factors. For addressing the interaction between factors we generated logistic regression model for both the subtype of distribution. When adjusted for other possible confounders through logistic regression model 'increased waist-to-hip ratio', table salt intake, psychosocial stress, presence of ECG abnormality and low fruit consumption persisted as significant predictor for Ischemic stroke. Hemorrhagic stroke model also showed a similar set of variables as predictor. Waist-to-hip ratio, table salt intake and low fruit consumption remained in the model. Additionally frequent fatty diet intake, hypertension and history of aspirin intake appeared as significant factor contributing to hemorrhagic stroke.

Smoking has been considered as a classical risk factor of all non-communicable diseases. In the Physicians Health Study two, ${ }^{17}$ the association of smoking with HS was approximately the same as that with IS. In a systematic review of 14 casecontrol and 11 cohort studies ${ }^{18}$ the relation was weak or not existent, whereas a recent pooled cohort of the Atherosclerosis Risk in Communities Study (ARIC) and Cardiovascular Health Study $(\mathrm{CHS})^{19}$ did not find any relation between HS and smoking. This study found smoking not to be associated with either type of stroke; in contrast smokeless tobacco appeared high in stroke patients, not statistically significant though as predictor of ischemic stroke. This form of tobacco use is widely prevalent particularly in females, and it shares almost half of the toll of tobacco consumption in our population. This form of tobacco is culturally accepted and it has prevention implication in a population with low income and education.

In our result low fruit and vegetables consumptions appeared as highly significant predictor of both the subtypes of stroke. In several articles, nutrients in fruit and vegetables such as dietary fiber, potassium, and antioxidants have been associated with reduced risk of cardiovascular diseases. ${ }^{20-22}$ Overall, these findings support a protective effect between consumption of fruits and vegetables against risk of stroke in both women and men. The constituents of fruits and vegetables potentially responsible for their apparent beneficial effects on risk of ischemic stroke include potassium, folate, and fiber; the associations between ischemic stroke and these nutrients have been evaluated in other reports. ${ }^{23,24}$ Dietary flavonoids present in fruits and vegetables have also been recently related to decreased risk of ischemic stroke. ${ }^{24}$ 
Study among Japanese men showed no statistically significant relation of stroke with dietary habit. They also found no relation between salt intake and the incidence of stroke. ${ }^{25}$ However, in our study table salt intake appeared as a significant predictor of both ischemic and hemorrhagic stroke. Hypertension is widely considered as a major contributor to stroke in the general population. Sacco et al. ${ }^{26}$ showed hypertension as a potent risk factor for stroke in general. However, in the studyhypertension posed almost 48 times more risk particularly for HS not in IS. Study on stroke subtype and hypertension support the differential across subtype of stroke ${ }^{27}$ but the factors behind the development of a particular stroke subtype in individual patients are not known. Salt intake may sometimes be considered as a intermediate factor for hypertension, which might affect association of hypertension.

A case control study in European population has shown obesity, hypertension and previous cardiac events or pathology associated with the highest population risk of ischemic stroke. They particularly focused on ECG pathology, presence of ischemic ECG changes are shown as highly associated with ischemic stroke, but less so with a history of IHD. ${ }^{28}$ In our data abnormal ECG change favored IS and was found to have no association with hemorrhagic stroke. Family history of CVD death and history of aspirin intake contributed to hemorrhagic stroke risk.

Another two major risk factors emerged in our data are obesity and stress. And physical activity level did not appear as risk factor. The WHR is a measure of abdominal obesity and a surrogate measure for visceral fat deposition. ${ }^{29}$ The importance of abdominal obesity as a risk factor in Bangladesh may be related to the recent rapid transition experienced in the region. Improvement in socioeconomic status was associated with increased life expectancy, urbanization, and obesity. These changes are evidenced in the high prevalence observed in our control group of abdominal obesity, sedentarism. Like our study waist-to-hip ratio was associated with increased risk of all stroke, and both ischemic and intracerebral hemorrhagic stroke also support our findings. Increased WHR was associated with a greater risk of stroke in men and women and in all race-ethnic groups. ${ }^{30}$

In general, physical activity is less in our population. However a distinct subgroup exists that is engaged in sedentary life style. Obesity and physical activity level in individual isoften regarded as reciprocal. Central obesity as proxy might have overridden the association of collinear attribute. in our data central obesity in terms of waist to hip ratio favored both ischemic and hemorrhagic stroke subtype. Psychosocial stress also appeared as a common predictor.

This study is the first case-control study aimed at identifying the relation between risk factors and hemorrhagic and ischemic stroke in Bangladeshi Population. The study was conducted in a tertiary care hospital serving an urban and suburban population in Dhaka, the capital city of Bangladesh. Since the cases are confirmed cases of stroke with or without having previous heart disease, the study avoids the problem of misdiagnosis associated with sole use of neuro-imaging as diagnostic criteria. We also used symptoms but there was chance of modification of risk factors by treatment of IHD. The problems associated with migration and acculturation also avoided because the study included Bangladeshi residents only; it unveiled the risk factors among individuals within a relatively homogeneous ethnic origin and geographic region, rather than between populations.

Our study may have some of the limitations inherent in a case-control design. The controls were taken carefully from admitted patient to avoid putative risk factor. Although controls from the community would have been ideal, hospitalbased controls were easier to recruit and generally belonged to the same population as hospital-based cases. Furthermore, all cases were defined prospectively with accepted criteria ${ }^{2}$ and all controls were carefully screened clinically to eliminate those who may have had subclinical disease. Without a prospective cohort study, it is difficult to assess the potential impact on risk factors, if any, of pre-existing silent IHD, changing risk factors among cases, and any treatment for diabetes mellitus and hypertension that patients may have received. However, such factors generally tend to reduce differences between cases and controls. Finally, the patients in this study are predominantly male and any extrapolation to women should be done cautiously. Our findings suggest that, hypertension (which important risk factors for stroke in most populations), central obesity and less physical activity, less fruit consumption are important across a wide range of values in Bangladeshi population.

Data from our study suggest treatment of hypertension, reduction of visceral obesity, reducing salt and adding more fruit and vegetables are likely to be key factors in the prevention of premature stroke in our population. Although this study did not produce any new or surprising results, the value of small hospital-based studies like this one lies in adding to the knowledge gap of the 
important role of stroke prevention by influencing the subtype specific risk factors.

\section{References}

1. Dobkin B. The economic impact of stroke. Neurology. 1995;45: 6-9.

2. Wade DT. Stroke: rehabilitation and long-term care. Lancet. 992;339:791-93.

3. Feigin VL. Stroke in developing countries: can the epidemic be stopped and outcomes improved? Lancet Neurol 2007; 6: 94-97.

4. Strong K, Mathers C, Bonita R. Preventing stroke: saving lives around the world. Lancet Neurol 2007; 6 : 182-87.

5. Shi F, Hart RG, Sherman DG, Tegeler CH. Stroke in the People's Republic of China. Stroke 1989; 20:1581-85.

6. Taylor TN, Davis PH, Torner JC, Holmes J, Meyer JW, Jacobson MF. Lifetime cost of stroke in the United States. Stroke 1996; 27:1459 -66.

7. Rahman KM. Risk factors \& clinical presentations--a study of eighty-five hospital admitted stroke cases. Mymensingh Med J. 2002; 11(2): 113-15

8. Andersen KK, Olsen TS, Dehlendorff C, Kammersgaard LP. Hemorrhagic and Ischemic Strokes Compared Stroke Severity, Mortality, and Risk Factors. Stroke 2009;40: 2068-72.

9. Meschia JF. Clinically translated ischemic stroke genomics. Stroke 2004; 35(1): 2735-39.

10. Franke CL, Swieten JCV, Algra A, Gijn JV. Prognostic factors in patients with intracerebral hematoma. $J$ Neurol Neurosurg Psychiatry. 1992;55: 653- 57.

11. Qureshi AI, Tuhrim S, Broderick JP, Batjer HH, Hondo $\mathrm{H}$, Hanley DF. Spontaneous intracerebral hemorrhage. N Engl J Med 2001; 344: 1450-60.

12. Ferro JM. Update on cerebral hemorrhage. J Neurol. 2006; 253: 985-99.

13. Badiuzzaman M, Mohammad FR, Bari MS, Alam MB, Ahsan HAMN. Prevalence of modifiable risk factors among stroke patients in tertiary care hospital in Dhaka. J Med 2009; 10: 18-21.

14. Jørgensen HS, Nakayama H, Raaschou HO, Olsen TS. Intracerebral hemorrhage versus infarction: Stroke severity, risk factors and prognosis. Ann Neurol 1995 38: 45-50.

15. Hajat C, Dundas R, Stewart JA, Lawrence E, Rudd AG, Howard R, Wolfe CDA. Cerebrovascular risk factors and stroke subtypes. Differences between ethnic groups. Stroke 2001; 32: 37-42

16. Liu XF, van Melle G, Bogousslavsky J. Analysis of risk factors in 3901 patients with stroke. Chin Med Sci 2005; 20: 35-39.

17. Song Y-M, Sung J, Lawlor D, Smith GD, Shin Y, Ebrahim S. Blood pressure, hemorrhagic stroke, and ischemic stroke: the Korean national prospective occupational cohort study. BMJ 2004; 328: 324-25.

18. Zia E, Hedblad BO, Pessah-Rasmussen H, Berglund G, Janzon L, Engstrom G. Blood pressure in relation to the incidence of cerebral infarction and intracerebral hemorrhage: hypertensive hemorrhage: debated nomenclature is still relevant. Stroke 2007;38:2681-85.

19. Ariesen MJ, Claus SP, Rinkel GJE, Algra A. Risk factors for intracerebral hemorrhage in the general population. A systematic review. Stroke 2003; 34: 2060-66.

20. Rimm EB, Stampfer MJ, Ascherio A, Giovannucci E, Colditz GA, Willett WC. Vitamin E consumption and the risk of coronary heart disease in men. $N$ Engl JMed 1993;328:1450-56

21. Khaw KT, Barrett-Connor E. Dietary potassium and stroke-associated mortality: a 12-year prospective population study. N Engl J Med 1987;316:235-40.

22. Knekt P, Reunanen A, Jarvinen R, Seppanen R, Hellovaara M, Arommaa A. Antioxidant vitamin intake and coronary mortality in a longitudinal study. Am J Epidemiol 1994;139:180-89.

23. Gaziano JM, Manson JE, Branch LG, Colditz GA, Willett WC, Buring JE. A prospective study of consumption of carotenoids in fruits and vegetables and decreased cardiovascular mortality in the elderly. Ann Epidemiol 1995;5:225-60.

24. Keli SO, Hertog MG, Feskens EJ, Kromhout D. Dietary flavonoids, antioxidant vitamins, and incidence of stroke: the Zutphen study. Arch Intern Med 1996; 156: $637-42$

25. Selhub J, Jacques PF, Bostom AG, D'Agostino RB, Wilson PW, Belanger AJ, et al. Association between plasma homocysteine concentrations and extracranial carotid-artery stenosis. N Engl J Med 1995;332:286-91.

26. Sacco RL, Boden-Albala B, Abel G, Lin IF, Elkind M, Hauser WA, et al. Race-Ethnic Disparities in the Impact of Stroke Risk Factors. Stroke 2001; 32(8): 1725-31.

27. Kagan A, Popper JS, Rhoads GG, Yano K. Dietary and other risk factors for stroke in Hawaiian Japanese men. Stroke 1985;16(3): 390-96.

28. Hajat C, Tilling K, Stewart JA, Lemic-Stojcevic N, Wolfe CDA. Ethnic Differences in Risk Factors for Ischemic Stroke. Stroke 2004; 35(7):1562-67.

29. Seung-HS, Ralph LS, Bernadette BA, Jian FC, John GP, Mitchell SE, Myunghee CP. Abdominal Obesity and Risk of Ischemic Stroke, The Northern Manhattan Stroke Study Stroke 2003;34:1586-92.

30. Kvist H, Chowdury B, Grangard V, Tylen V, Sjostrom L. Total and visceral adipose tissue volumes derived from measurements with computed tomography in adult men and women: predictive equations. Am $J$ ClinNutr1988: 48 1351-61 